\title{
Osteobiografía de dos individuos inhumados durante la Campaña del Desierto: el caso del sitio Chimpay (Argentina)
}

\author{
Alejandro SERNA \\ CONICET. División Arqueología, Museo de La Plata, Argentina \\ alejandroserna@hotmail.com.ar \\ Luciano Prates \\ CONICET. División Arqueología, Museo de La Plata, Argentina \\ Leandro LUNA \\ CONICET. Museo Etnográfico J. B. Ambrosetti, Buenos Aires, Argentina
}

Recibido: 28 de julio de 2014

Aceptado: 25 de enero de 2016

\section{RESUMEN}

El sitio Chimpay se encuentra en el valle medio del río Negro, pocos kilómetros al oeste de la isla de Choele Choel, en el centro-sur de la República Argentina. Se trata de un contexto de fines del siglo XIX conformado por el entierro doble de un hombre y una mujer adultos, asociados con un abundante y diverso acompañamiento funerario de origen europeo e indígena, que sugiere que habrían gozado de cierta jerarquía y que el hombre habría ostentado un cargo militar en el ejército argentino. A fin de esclarecer este complejo ritual funerario, el presente trabajo parte de un enfoque osteobiográfico y busca avanzar en la identificación biológico-poblacional de los individuos e inferir condiciones generales de vida.

Palabras clave: Siglo XIX, bioarqueología, osteobiografía.

\section{Osteobiography of Two Individuals Buried During the Desert Campaign: The Case of the Chimpay Site (Argentine)}

\begin{abstract}
The Chimpay site is located in the Negro River middle valley, a few miles west from Choele Choel Island, in the south-center of Argentina. It is a late nineteenth century context that consists in a double burial of a man and a woman associated with a rich and diverse european and indigenous funeral offering, suggesting that they had a hierarchical social position and that the male would have had an Argentine military rank. In order to clarify this complex funeral ritual, the paper develops an osteobiographical approach and aims to give information about biological identification of the individuals and to infer their general living conditions.
\end{abstract}

Key words: Nineteenth century, bioarchaeology, osteobiography.

Sumario: 1. Introducción. 2. Materiales y métodos. 3. Resultados. 4. Discusión. 5. Consideraciones finales. 6. Referencias bibliográficas.

\section{Introducción}

Desde mediados del siglo XIX, han sido registrados centenares de restos humanos en la cuenca del río Negro, en su mayoría correspondientes a momentos prehispánicos (véase síntesis en Prates y Di Prado 2013). Este trabajo se centra en el estudio bioarqueológico del sitio Chimpay, cuyo contexto lo ubica en el núcleo del avance del Estado argentino sobre las poblaciones indígenas de Norpatagonia a finales del 


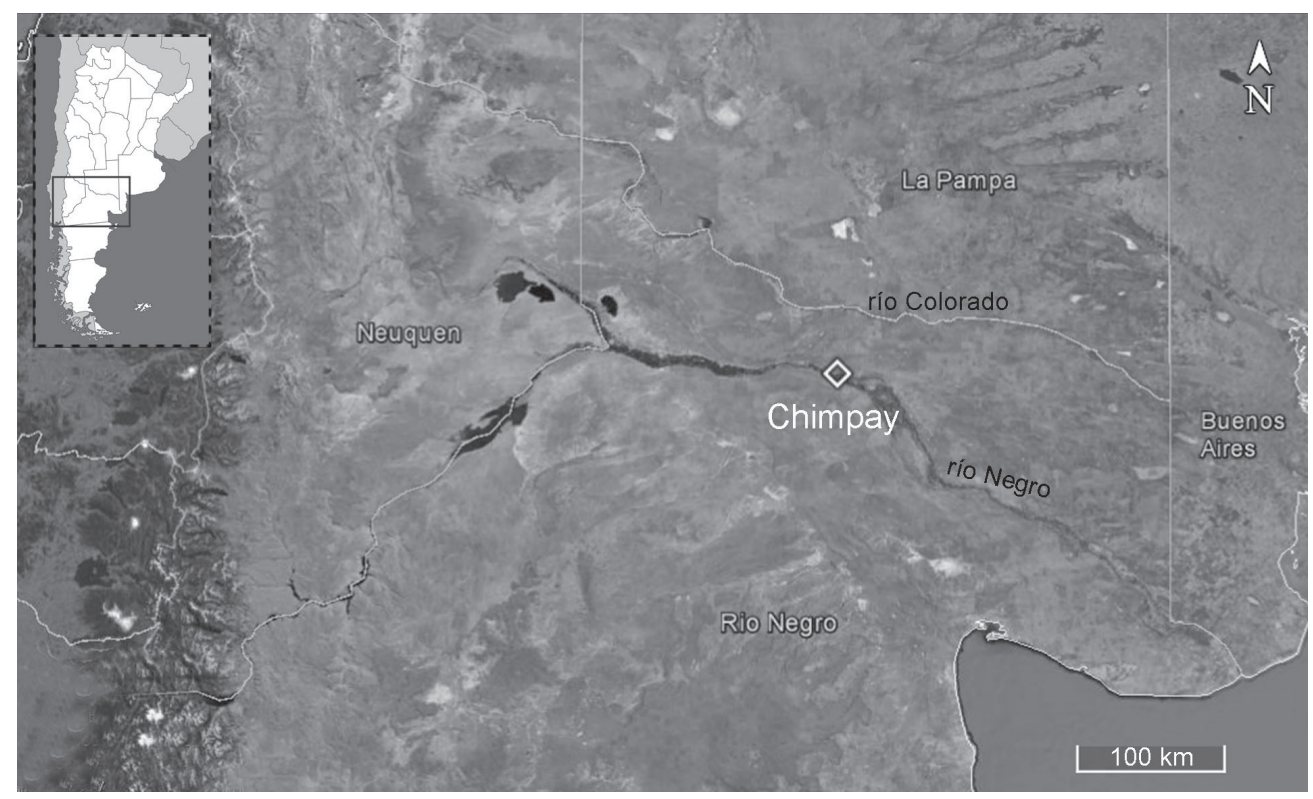

Figura 1: Ubicación del sitio arqueológico Chimpay.

siglo XIX (Prates et al. 2016). El hallazgo se produjo accidentalmente durante la nivelación del terreno con maquinaria agrícola y, gracias a la gestión del Sr. Daniel Cabaza, Director del Museo Paleontológico Municipal de Lamarque, se realizó la denuncia a la Secretaría de Cultura de Río Negro. Los posteriores trabajos de rescate se efectuaron por requerimiento de dicho organismo.

El sitio Chimpay se encuentra ubicado en el sector norte del valle medio del río Negro, 2,6 km al sudeste de la localidad homónima y $c a .20 \mathrm{~km}$ río arriba de la

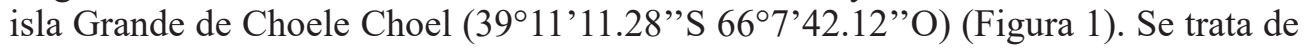
un contexto mortuorio en el que se recuperaron dos esqueletos humanos muy bien preservados. Los individuos conformaban un entierro primario doble asociado a un abundante y diverso acompañamiento funerario en el que coexisten objetos de origen indígena y europeo (Prates et al. 2016). Junto al Individuo 1 se recuperaron restos de indumentaria que sugieren que fue sepultado portando un uniforme militar argentino de fines del siglo XIX. Además, estaba acompañado por diversos objetos, como un prendedor confeccionado con una moneda de plata del siglo XVIII, una bandolera de arma de fuego o sable, botellas de ginebra holandesa, un costillar de oveja, un recipiente metálico y fragmentos de cucharas. El Individuo 2 estaba acompañado por más de 7.000 cuentas vítreas de diferentes formas, tamaños y colores, una jarra con asa de loza, una navaja cortaplumas, una tortera lítica decorada, varios accesorios de metal, fragmentos de textil y algunos restos faunísticos.

Son varios los ejemplos conocidos a través de la arqueología, y principalmente de la etnohistoria, sobre la complejidad y diversidad de los rituales funerarios durante el período de avance militar del Estado Nacional sobre las poblaciones indígenas (e.g. González 1979). Teniendo en cuenta esta complejidad y las particularidades del 
contexto, su estudio requiere de diversas vías de análisis. Desde la bioarqueología, este trabajo se centra en el estudio de los individuos y busca definir sus trayectorias de vida e interpretarlas en el marco del contexto socio-histórico particular en el que vivieron (Larsen 2000). Los objetivos principales de este artículo son avanzar en la identificación biológico-poblacional, a fin de determinar si se trata de individuos biológicamente adscritos a poblaciones nativas o criollas e inferir sus condiciones generales de vida.

\section{Materiales y métodos}

Los dos individuos que conforman la muestra se encuentran anatómicamente completos y muy bien preservados. Para la determinación del sexo se consideraron los caracteres morfológicos clásicos de elementos dimórficos como los coxales y el cráneo (Buikstra y Ubelaker 1994; White y Folkens 1991). La edad probable de muerte se estimó a partir del relevamiento de la sínfisis púbica, la superficie auricular y la zona preauricular (Buikstra y Ubelaker 1994; Lovejoy et al. 1985).

Para establecer una posible filiación biológica, se analizaron las variables discretas dentales propuestas en los protocolos de Scott y Turner (2000) y Turner et al. (1991). Considerando la influencia multigénica en el control ontogenético de la dentición y el acotado lapso de su desarrollo en comparación con la alta influencia del medioambiente en el crecimiento y desarrollo esqueletal (Guatelli-Steinberg et al. 2001; Scott 1980), estos rasgos morfológicos presentan variaciones heredables y, tomados en conjunto, pueden contribuir a identificar patrones de herencia y caracterizar afinidades biológicas entre poblaciones (Hillson 1996; Sperber 2004; Ubelaker 1982). En el Individuo 1 fue posible relevar la mayoría de los dientes (todos excepto la dentición posterior superior izquierda, ausentes postmortem), mientras que para el Individuo 2 sólo fue posible analizar tres dientes inferiores (segundo incisivo izquierdo, segundo premolar izquierdo y canino derecho; la mayoría de los restantes están ausentes antemortem y algunos postmortem).

La totalidad de los restos recuperados de ambos individuos se analizaron macroscópicamente a fin de identificar indicadores de estrés mecánico, salud bucal y lesiones traumáticas, infecciosas, metabólicas o congénitas (Ortner 2003; Waldron 2009). Para relevar el estrés mecánico se observaron las superficies articulares en busca de indicios de degeneración articular -osteoartrosis- y alteración de las zonas de inserción muscular-cambios entésicos- (e.g. Jurmain et al. 2012; Rando y Waldron 2012; Waldron 2009). Para el relevamiento de la osteoartrosis, se siguieron los criterios propuestos por Neves (1984) y Rodrigues Carvalho (2004). Se observaron las superficies articulares y se las clasificó en base al grado de afección como leve (presencia de afilamiento incipiente en los márgenes articulares y/o exóstosis menores a $1 \mathrm{~mm}$; puede advertirse porosidad subcondral), moderada (presencia de labiaciones de entre 1 y $2 \mathrm{~mm}$ y aumento de la porosidad) y severa (presencia de proyecciones osteofíticas de más de $2 \mathrm{~mm}$; mayor porosidad y/o eburnado). Para el relevamiento de las zonas entésicas se siguieron los criterios utilizados por Hawkey y Merbs (1995), calificando las exóstosis (proliferativas) como leves $(<2 \mathrm{~mm})$, moderadas (entre 2 y $5 \mathrm{~mm}$ ) 


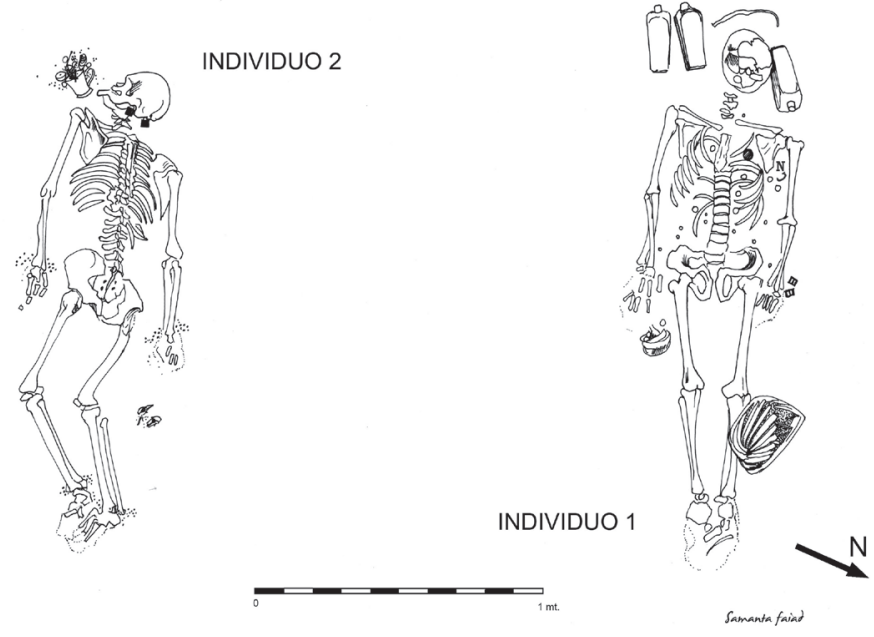

Figura 2: Esquema en planta de los individuos expuestos tras la excavación (tomado y modificado de Prates et al. 2016).

y severas ( $>5 \mathrm{~mm}$ ) y las lesiones de estrés (erosivas) como leves (surco superficial), moderadas (entre 1 y $3 \mathrm{~mm}$ de profundidad y $<5 \mathrm{~mm}$ de extensión) y severas ( $>3 \mathrm{~mm}$ de profundidad y $>5 \mathrm{~mm}$ de extensión). Aunque en este trabajo se considera que la degeneración articular y los cambios entésicos son indicadores de estrés mecánico, su etiología también está vinculada a factores tales como la edad, el sexo, el tamaño corporal, las predisposiciones genéticas y los desarrollos patológicos (Jurmain 1999; Weiss y Jurmain 2007; Wilczak 1998). De todas maneras, la demanda mecánica corporal es un factor importante y útil para inferir patrones generales de uso del cuerpo (e.g. Molnar et al. 2011; Villotte et al. 2010). Por último, se examinaron la zona alveolar y las piezas dentales en busca de indicadores macroscópicos de salud bucal considerando la pérdida dental antemortem, el cálculo dental, la enfermedad periodontal, el desgaste dental, la caries y las lesiones periapicales (Hillson 1996; Lukacs 1989; Molnar 1971; Ogden 2008; Smith 1984).

\section{Resultados}

\subsection{Individuo 1}

El Individuo 1 estaba dispuesto en posición decúbito dorsal extendida con los miembros superiores paralelos al tronco (Figura 2). El cráneo no presenta deformación artificial y está fracturado postmortem en su sector izquierdo. Del esqueleto postcraneal sólo faltan algunas falanges y el radio izquierdo. En general la preservación de los elementos ( $\mathrm{n}=148)$ es óptima; todos están completos en más de un $75 \%$ y cuatro (2,7\%; húmero y cúbito izquierdos, y escápula y clavícula derechas) exhiben una leve tinción de óxido de cobre derivada del deterioro de algunos de los materiales culturales asociados.

Es un individuo masculino adulto joven ( 25 a 35 años aproximadamente) en el momento de la muerte. En la dentición se registraron tres rasgos morfológicos re- 
Figura 3: Individuo 1. a) Caninos superiores en forma de pala e incisivos superiores con forma de pala y surcos de interrupción; b) Incisivo central superior izquierdo con forma de pala (detalle de la vista lingual a la izquierda) y doble pala (detalle de la vista labial a la derecha); c) Nódulos de Schmorl en T7 y T6; d) Exostosis en el aspecto medial del olécranon derecho; e) Lesión de estrés ubicada en la tuberosidad costal de la clavícula derecha.

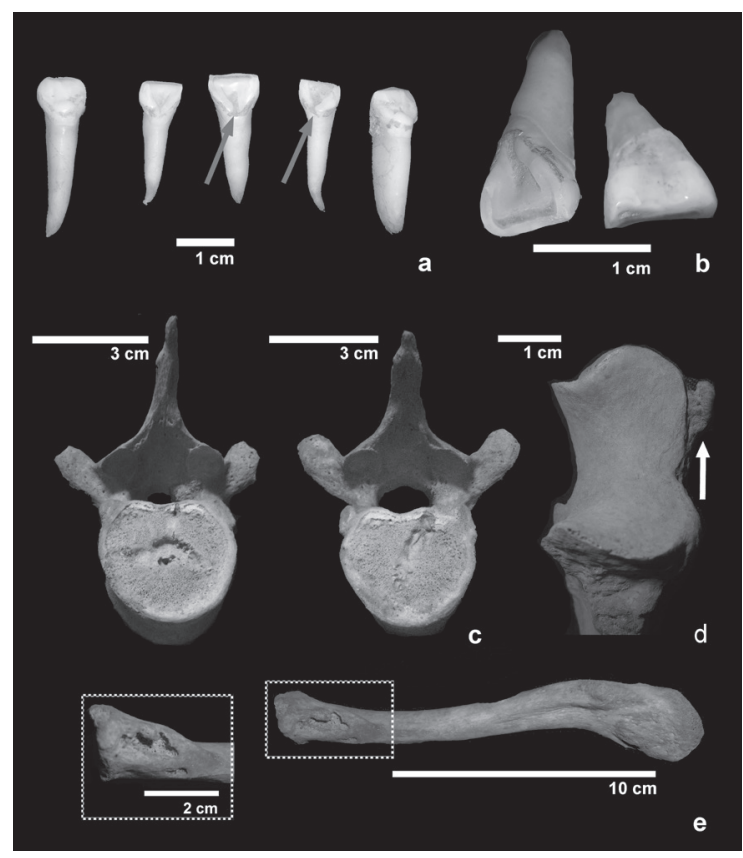

levantes como indicadores de filiación biológico-poblacional: a) dientes en forma de pala (shoveling), identificados en toda la dentición anterior (incisivos y caninos superiores e inferiores) y en grados extremos (categorías 4 a 6; Scott y Turner 2000); b) dientes en forma de doble pala (double-shoveling) en incisivos centrales y laterales superiores, también en grado máximo (categorías 4 y 5; Scott y Turner 2000) y c) surcos de interrupción (interruption grooves) mesiales largos en los incisivos superiores (Figuras $3 a \mathrm{y} b$ ).

En lo que respecta a los marcadores de estrés mecánico, para la osteoartrosis se relevaron 257 superficies articulares. Un 6,2\% $(\mathrm{n}=16)$ exhibe alteraciones artropáticas: el cúbito derecho presenta señales severas en su epífisis distal y el resto de las lesiones son leves y se distribuyen en todas las regiones de la columna vertebral, mayoritariamente en las facetas intervertebrales. Se observan lesiones en forma de depresión sobre varios cuerpos de vértebras torácicas y, con menor desarrollo, en dos lumbares $(\mathrm{n}=7 ;$ T6, T7, T10, T11 y L3 fueron afectadas sobre la superficie inferior, L4 sobre la superior y T8 sobre ambas). Todas ellas son asignables a un trastorno conocido como Nódulo de Schmorl o hernia intraesponjosa (Waldron 2009) (Figura 3c). En lo referente a las entesoexóstosis, el cúbito derecho registra una manifestación severa sobre la parte medial del olécranon (inserción del tríceps braquial) (Figura 3d). Ambas líneas ásperas femorales (inserción de aductores) presentan una manifestación leve. Además, se observan lesiones de estrés localizadas en la tuberosidad costal de ambas clavículas (inserción del ligamento costoclavicular), incipiente en la izquierda y severa en la derecha (Figura 3e).

Respecto de la dentición, el maxilar presenta varias pérdidas postmortem (toda la dentición posterior izquierda y el incisivo central derecho). Los restantes dientes per- 
manecen en oclusión, con un desgaste plano y leve (grados 3 y 4; Smith 1984) y una mínima exposición de la dentina. Todos tienen cálculo supragingival adherido, menor en los dientes posteriores y mayor en los anteriores (grados 1 y 2; Brothwell 1981). En la mandíbula se registra la pérdida antemortem del tercer molar izquierdo. La dentición posterior presenta un desarrollo leve de cálculo (grado 1), mientras que en la anterior es más intenso (grado 3). Los dientes presentan un desgaste plano y relativamente bajo (grados 3 y 4) con exposición leve de la dentina, exceptuando los primeros molares, cuyo desgaste es más severo (grado 5). Se identificó una caries oclusal en las fisuras del tercer molar inferior derecho que afectó exclusivamente al esmalte. No se observaron lesiones periapicales, alteraciones periodontales, indicadores de trauma ni evidencias de enfermedades metabólicas, infecciosas o congénitas.

\subsection{Individuo 2}

El Individuo 2 fue depositado en posición decúbito ventral extendida, con los miembros superiores paralelos al tronco y los inferiores levemente flexionados (ver Figura 2). El cráneo se recuperó entero y tampoco presenta deformación artificial. Del esqueleto post-craneal sólo faltan algunas falanges de manos y pies. La preservación de los elementos $(n=145)$ es óptima (completitud $>75 \%)$; unos pocos $(n=8 ; 5,5 \%$; radios y cúbitos, tercera y cuarta vértebras cervicales, cráneo y mandíbula) muestran tinción de óxido de cobre derivada del deterioro de algunos de los materiales culturales asociados. Se trata de un individuo femenino adulto medio (40 a 49 años aproximadamente). En este caso no pudo realizarse el análisis morfológico de los dientes porque sólo se recuperaron tres elementos que están recubiertos con una densa capa de cálculo.

En cuanto a los indicadores de estrés mecánico, se relevaron 261 carillas articulares; el 62,8\% $(n=164)$ presenta alteraciones que comprometen la articulación temporomandibular (ATM), la columna vertebral, ambas cinturas y todo el esqueleto apendicular. La ATM está afectada sobre los cóndilos mandibulares, de forma leve en el derecho y severa en el izquierdo, sin formación de osteofitos pero con desgaste masivo y porosidad. La lesión del cóndilo izquierdo podría ser compatible con una osteocondritis (Waldron 2009). Los cóndilos occipitales presentan osteofitosis leves. Todas las vértebras están afectadas, a excepción del atlas; la manifestación más frecuente es la osteofitosis, tanto en las facetas articulares intervertebrales como en los cuerpos, con predominio del grado severo (Figura 4a). En algunos casos, estas formaciones también están acompañadas por porosidad y eburnación. La mayor parte de los cuerpos vertebrales $(n=39 ; 83 \%)$ fue afectada por osteofitos moderados y severos; en algunos casos forma labiaciones sobre los márgenes anteriores. Se detectaron también algunos casos de porosidad $(n=11 ; 23 \%)$ distribuidos en las tres regiones de la columna y de eburnación $(n=4 ; 9 \%)$ en las regiones cervical y lumbar. Las facetas articulares superiores e inferiores están afectadas en un alto porcentaje $(n=80 ; 82 \%)$ por osteofitos (leves: $n=44,45 \%$; moderados: $n=11,11 \%$; severos: $n=25,26 \%$ ), incluyendo en varios casos deformación de los contornos articulares y, en menor medida, por porosidad $(n=36 ; 37 \%)$ y eburnación $(n=24 ; 24 \%)$. La cintura escapular presenta osteoartrosis severa en la cavidad glenoidea izquierda, moderada en la derecha y leve 
Figura 4: Individuo 2. a) vertebras C5, T10 y L5 con señales severas de osteoatrosis; b) Proyección ósea y eburnación en la rótula derecha; c) Porosidad y eburnación en el cóndilo lateral del fémur derecho; d) Faceta lateral en el mismo sector; e) Exostosis en el sector anterior del trocánter mayor del fémur izquierdo; f) Desarrollos óseos palmares en diáfisis de falanges proximales; g) Faceta dorsal en epífisis proximal de primera falange proximal; h) Mandíbula con pérdidas dentales antemortem y postmortem; i) Excrecencia dorsal en cabeza del tercer metatarsiano derecho; j) Maxilar con pérdida dental antemortem total.

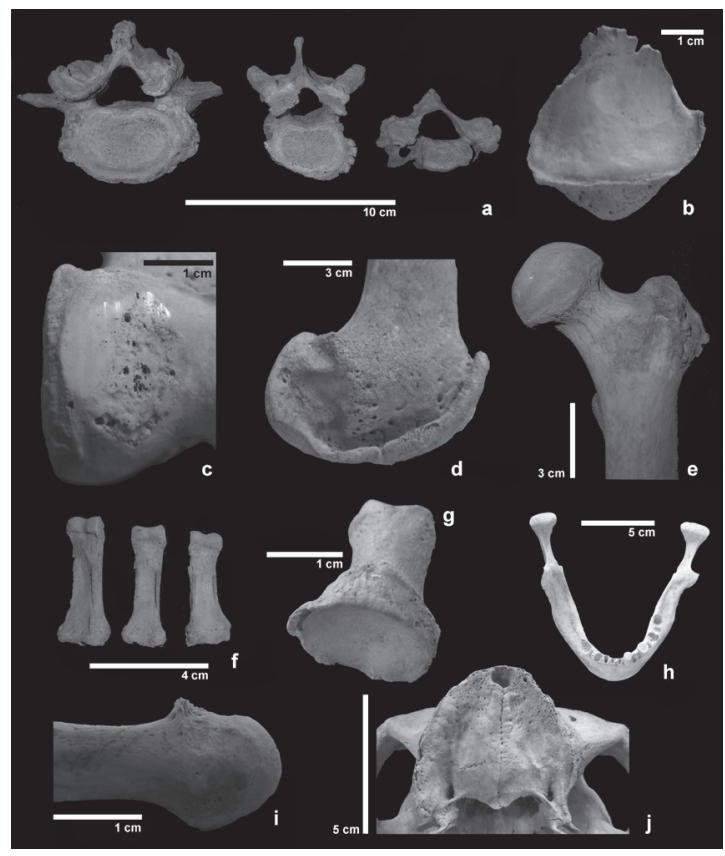

en las cabezas de los dos húmeros. Ambos codos exhiben un grado leve, tanto en las articulaciones distales de los húmeros como en las proximales de los radios y de los cúbitos. Las muñecas están severamente afectadas en las articulaciones distales de los radios, con manifestaciones más intensas en la izquierda y los cúbitos presentan osteofitos leves, aunque el izquierdo exhibe además señales de eburnación. La mayor parte de los metacarpos presenta proliferaciones leves y moderadas en su base, mientras que algunas falanges exhiben únicamente osteofitosis leves. La articulación coxofemoral presenta osteoartrosis moderada en el acetábulo izquierdo y severa en el derecho, mientras que las cabezas femorales están completamente exentas. La rótula y los cóndilos del fémur izquierdo exhiben manifestaciones leves y una lesión en forma de depresión con porosidad y remodelación sobre el sector anterior del cóndilo lateral, compatible con una osteocondritis (Waldron 2009). La articulación del lado derecho está más afectada, con manifestaciones osteofíticas severas, porosidad y eburnación, en el fémur y en la rótula (Figuras $4 \mathrm{~b}$ y 4c). Además, se registró en los cóndilos una faceta de forma semilunar proyectada hacia los laterales (Figuras 4d). En las articulaciones de los pies, ambos calcáneos muestran manifestaciones leves; el primer metatarsiano derecho presenta eburnación y algunas falanges proyecciones óseas leves. Además, se observan excrecencias óseas sobre la superficie dorsal de la cabeza del tercer metatarsiano derecho y una pequeña faceta definida por una excrecencia ósea dorsal que se extiende hacia el extremo distal en las primeras falanges proximales y la segunda proximal derecha (Figuras 4f, $4 \mathrm{~g}$ y $4 \mathrm{i}$ ).

En las zonas de inserción muscular se registraron numerosas proliferaciones óseas. Ambos húmeros presentan exostosis leves en la corredera bicipital (zona de inserción del pectoral mayor) y en la tuberosidad deltoidea (zona de inserción del deltoides). El 


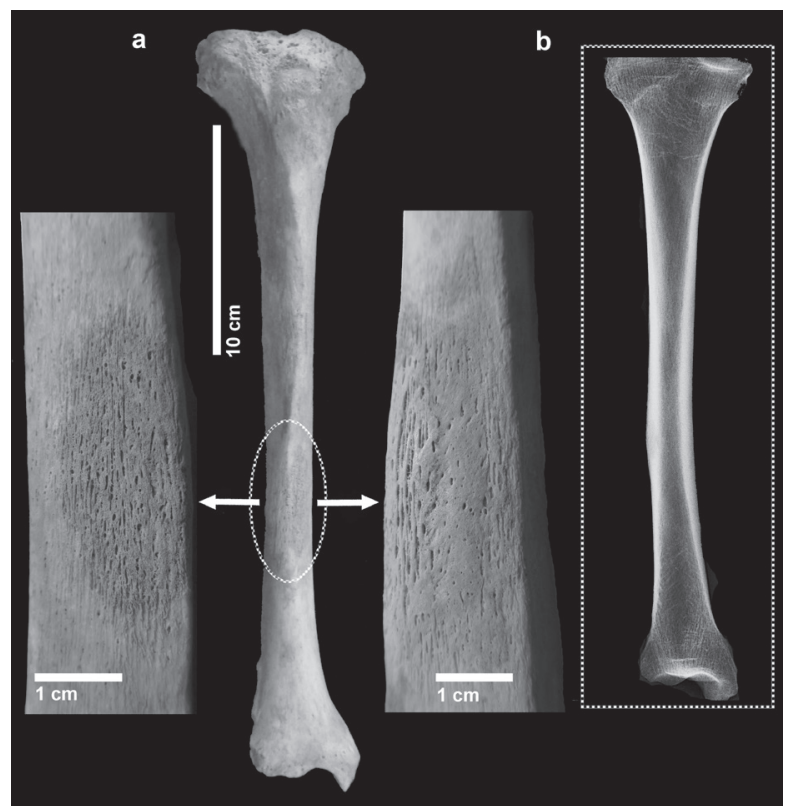

Figura 5: Individuo 2. a) Tibia derecha con detalle de la reacción lateral (izquierda) y medial (derecha); b) Imagen radiográfica.

radio derecho exhibe una exostosis moderada en la tuberosidad radial (zona de inserción del bíceps braquial). Ambos cúbitos presentan exostosis en la parte superior del olécranon (zona de inserción del tríceps braquial), moderada en el izquierdo y leve en el derecho. La mayoría de las falanges de las manos muestra excrecencias óseas leves, que sobresalen ventralmente de los bordes lateral y medial de la diáfisis (zona de inserción de los flexores superficial y profundo) (ver Figura 4f). En los miembros inferiores, los coxales manifiestan excrecencias óseas leves (en el izquierdo) y moderadas (en el derecho) a lo largo de la cresta ilíaca (zona de inserción del músculo oblicuo externo del abdomen). Ambos fémures tienen exostosis moderadas en la cresta glútea y fascia lata (zona de inserción del glúteo mayor), severa en el borde anterior del trocánter mayor izquierdo y leve en el derecho (zona de inserción glúteo menor) (Figura 4e).

En lo que respecta a la salud bucal, el individuo presenta pérdida dental antemortem total de las piezas superiores, con reabsorción alveolar completa (Figura 4j). La mandíbula presenta tres dientes en los alvéolos (segundo incisivo y primer premolar izquierdos y canino derecho), varias pérdidas postmortem (primer incisivo, canino y segundo premolar izquierdos y ambos incisivos y primer premolar derechos), alvéolos en proceso de obliteración (segundo premolar y molares izquierdos) y otros completamente obliterados (segundo premolar y molares derechos), indicando una pérdida antemortem masiva (Figura 4h). Los dientes tienen un desgaste plano y severo (grados 5 y 6; Smith 1984), con amplia exposición de la dentina, y están recubiertos por una densa y amplia capa de cálculo supragingival (grado 3; Brothwell 1981). El deterioro postdepositacional de los alveolos presentes impidió el relevamiento adecuado de las alteraciones periodontales. No se registraron caries, lesiones periapicales ni otras alteraciones patológicas en el sector bucal. 
Por último, se destaca la presencia de una lesión en el tercio medio anterior de la tibia derecha. La cara medial presenta hueso nuevo de aspecto liso y textura suave que engrosa la zona de la lesión, y alrededor de la misma, depositación de tejido irregular reticular y de textura rugosa (i.e. tejido de woven). En la cara lateral se identifica la depositación de tipo woven, uniforme y de forma ovoidal, que entra en contacto con la otra lesión. Estas manifestaciones patológicas indican un foco primario (neoformación más densa) con una ramificación posterior más abarcativa. Las observaciones radiográficas muestran un discreto hematoma osificado derivado de una osteítis, con engrosamiento exclusivo a nivel cortical, y descartan un compromiso del endostio (Van der Merwe et al. 2010) (Figura 5).

\section{Discusión}

\subsection{Filiación poblacional del Individuo 1}

El Individuo 1 posee atributos dentales que permiten inferir aspectos vinculados con su filiación biológico-poblacional. Se identificaron manifestaciones intensas de diente en forma de pala, doble pala y surcos de interrupción (ver Figura 3). Estos rasgos suelen presentar frecuencias elevadas $(>80 \%$ para los primeros y $>50 \%$ para los surcos de interrupción) en poblaciones asiáticas y amerindias, y muy bajas $(<15 \%)$ en poblaciones europeas y africanas (Hrdlicka 1920; Scott y Turner 2000; Turner et al. 1991, entre otros). Además, no se observaron otras variables frecuentes en poblaciones europeas ( $>55 \%$ aproximadamente) e infrecuentes en asiáticas y amerindias $(<15 \%$ aproximadamente), como la cúspide de Carabelli, el parastyle, el protostílido y las cúspides extranumerarias (Scott y Turner 2000; Turner et al. 1991). La elevada prevalencia de keilomorfismo (forma de pala) representa uno de los principales componentes del denominado Complejo Dental Mongoloide u Oriental (Hanihara 1968), patrón típico de los nativos americanos identificado en numerosas muestras arqueologías americanas (e.g. Bollini et al. 2006; Devoto et al. 1968). Estos resultados indican que la posibilidad de pertenencia a un linaje nativo americano para este individuo es alta. Si bien una asignación poblacional conclusiva sólo puede llevarse a cabo desde una perspectiva poblacional y teniendo en cuenta la posibilidad de mestizaje, la probabilidad de identificar una configuración de rasgos como la relevada en este caso es muy baja para muestras europeas y no se correspondería con las tendencias observadas en investigaciones comparativas megaescalares (Scott y Turner 2000).

\subsection{Estrés mecánico en el Individuo 1}

Los resultados muestran escasas prevalencias y leve desarrollo de osteoartrosis, concentrada en los sectores cervical y lumbar de la columna vertebral. En contraste con esta tendencia, es alta la prevalencia de nódulos de Schmorl en el último segmento de la región torácica y en la región lumbar. Estas hernias, formadas por la protrusión del núcleo pulposo en el cuerpo vertebral (Ortner 2003), suelen aparecer progresivamente en individuos adultos de edad avanzada, mientras que en jóvenes general- 
mente ocurre como producto de traumas o de fuerzas de sobrecarga vertical crónicas aplicadas sobre la columna (Bolm-Audorff 1992; Edynak 1976; Pfirrmann y Resnick 2001). Teniendo en cuenta que las lesiones en la zona toracolumbar pueden vincularse a la actividad recurrente de monta a caballo (e.g. Hessler et al. 2012; Wentz y de Grummond 2009) y que el contexto histórico del sitio se asocia con las jefaturas ecuestres de los siglos XVIII y XIX (Mandrini 2008; Nacuzzi 1998), es razonable plantearla como uno de los posibles factores causales del trastorno. En el resto del esqueleto, la única señal de compromiso articular se registra en la muñeca derecha. Si bien resulta difícil establecer la etiología de estas lesiones, podría considerarse la posibilidad de que se deba a la realización de actividades manuales que requieren de firmeza y movilidad. Teniendo en cuenta los cambios entésicos, los miembros superiores registran lesiones de estrés en ambas clavículas, indicando un uso recurrente e intenso de los hombros (Mann y Murphy 1990). Este tipo de lesiones puede estar relacionado con actividades tales como la carga de pesos sobre los hombros, tareas de arado y actividades acuáticas, entre otras (e.g. Galera y Garralda 1993). Además, se infiere un uso exigido del tríceps braquial del cúbito derecho, vinculado con actividades de flexo-extensión del brazo (Capasso et al. 1999). En los miembros inferiores se identifica un desarrollo importante de las zonas de inserción de los aductores en ambos fémures; el contexto socio-histórico permite aquí también considerar la monta (e.g. Reinhard 1993; Üstündaĝ y Deveci 2011) entre las hipótesis más plausibles.

\subsection{Salud bucal del Individuo 1}

El Individuo 1 presenta un estado de salud bucal bueno con sólo una pérdida antemortem. Su dentición se caracteriza por la adherencia de cálculo en las coronas, principalmente en las piezas anteriores. Además, exhibe un desgaste homogéneo leve y plano atribuible al estrés mecánico producido por la incorporación de partículas abrasivas en el procesamiento de los alimentos consumidos (e.g. Cane 1989; Powell 1985).

\subsection{Filiación poblacional del Individuo 2}

La escasez de dientes y el grueso recubrimiento de cálculo no permitieron realizar el análisis de variables discretas para inferir filiación biológico-poblacional. Sin embargo, existen varios indicadores contextuales que sugieren una posible filiación cultural mapuche: la posición de los cuerpos orientada hacia el O-SO y la asociación del esqueleto con adornos corporales de cobre (e.g. aros trapezoidales y casquetes de cobre) y con una tortera para hilado (Prates et al. 2016).

\subsection{Estrés mecánico en el Individuo 2}

El Individuo 2 presenta altas prevalencias de lesiones osteoartrósicas y cambios entésicos en todo el esqueleto. Si bien se considera que el principal factor que provoca las degeneraciones articulares es el estrés mecánico (Jurmain 1990; Larsen 2000), 
hay que tener cuenta su etiología multifactorial (Weiss y Jurmain 2007). En este caso es probable que la edad haya incidido en los trastornos articulares y cambios entésicos, actuando en forma conjunta con el estrés de las actividades cotidianas (Jurmain 1990). Respecto de las afecciones osteoartrósicas, la registrada en la mandíbula probablemente sea consecuencia de la exigencia masticatoria resultante de la masiva pérdida dental (Hodges 1991; Merbs 1983). Esto es compatible con la osteocondritis del cóndilo izquierdo, en cuyo lado el individuo presenta dos de los tres dientes activos y que debió ser la zona de masticación sometida a mayores esfuerzos mecánicos. Por otra parte, la severa y extensiva afección articular de la columna puede asociarse con la aplicación de sobrecargas desde posturas que implicaron flexiones forzadas durante períodos prolongados (e.g. transporte de carga sobre la espalda y/o cabeza, actividades de molienda o posturas de hilado con huitral; Capasso et al. 1999; Knüsel 2000; Merbs 1983; Ortner 2003). Los miembros superiores se vieron afectados con mayor intensidad en el hombro izquierdo y de igual manera a nivel del codo, muñeca y mano. Este tipo de degeneración podría vincularse con diversas actividades (e.g. levantamiento de cargas, tareas hortícolas o la elaboración de cerámica; Lieverse et al. 2007). Algunos investigadores postulan que el uso de morteros puede resultar también en lesiones bilaterales del codo (Molleson 1994). En el caso de las falanges, la degeneración podría explicarse por el uso recurrente y exigido de los dedos en actividades repetitivas de precisión (e.g. hilado; Hadler 1977). Las articulaciones de los miembros inferiores están afectadas a nivel de la cadera, rodilla y tobillo-pie, sobre todo el miembro derecho. Aunque es frecuente que se vincule la degeneración en la cadera con factores hormonales y etarios, y en la rodilla con la intensidad de las actividades, es probable que las tareas que impactaron sobre éstas hayan influido también en la cintura pélvica (Bridges 1992; Jurmain 1980; Weiss y Jurmain 2007). La afección generalizada en los miembros inferiores podría relacionarse con intensas actividades deambulatorias (e.g. acarreo y transporte de cargas pesadas sobre terrenos dificultosos; Capasso et al. 1999; Jurmain 1980; Lieverse et al. 2007), aunque en este caso uno de los miembros está considerablemente más afectado que el otro y no se detectaron prácticamente lesiones en los pies. Además, varios elementos sugieren que el individuo habría realizado actividades que implicaban el acuclillamiento y/o arrodillamiento en forma reiterada, prolongada e intensa, ejerciendo mayor presión sobre el miembro derecho (e.g. Kennedy 1989; Martin 1932; Trinkaus 1975). El fémur izquierdo presenta osteocondritis producto de la hiperflexión femoro-tibial (Capasso et al. 1999), considerada como un marcador postural asociado al arrodillamiento (Lai y Lovell 1992; Ubelaker 1979). En el derecho, la formación de las facetas semilunares en los laterales de ambos cóndilos ha sido asociada a la presión de tendones durante la flexión de la rodilla (Kostick 1963; Martin 1932). Además, el cóndilo lateral femoral y la faceta lateral de la rótula presentan eburnación con estrías muy definidas (ver Figuras $4 \mathrm{~b}$ y c), evidenciando el intenso estrés mecánico y direccional sufrido por la articulación (Ortner 2003). La proyección osteofítica de la rótula presenta también estrías debido a que actuó como una superficie de ampliación de la faceta rotuliana original. Finalmente, las alteraciones registradas en los metatarsos y falanges podrían indicar la hiperdorsoflexión del pie desde una postura de rodillas o cuclillas. Una actividad probable que puede reunir estos indicadores tan marcados 
junto con la degeneración del otro miembro, es la actividad de molienda (Molleson 1994, 2007; Ubelaker 1979). Durante su realización, los pies probablemente fueron flexionados utilizando los primeros metatarsianos para hacer palanca (Murillo 2002) y las rodillas actuaron como pivote para realizar los movimientos lineales en sentido anteroposterior (Molleson 1994).

De acuerdo con el análisis de las zonas entésicas, se identificó un predominio del desarrollo muscular de los miembros superiores. Se observa bilateralmente en el húmero el correlato del uso intenso del pectoral mayor y del deltoides, en el cúbito del tríceps braquial y en el radio derecho del bíceps braquial. Todos ellos en conjunto actúan en acciones de flexo-extensión, aducción, abducción, rotación del brazo y movimientos en supinación del antebrazo. Este desarrollo extenso y bilateral de la musculatura de los miembros superiores ha sido vinculado con movimientos lineales, intensos y repetitivos (e.g. molienda, el remo, el cuereo, etc.; Capasso et al. 1999; Eshed et al. 2004; Hawkey y Merbs 1995). Las proliferaciones óseas en las falanges de las manos sugieren el uso reiterado e intensivo de los flexores de los dedos y la muñeca, posiblemente vinculado con prensión y movimientos continuos y precisos (e.g. molienda, tejido, etc.; Capasso et al. 1999; Freivalds 2004). Ambos miembros inferiores presentan un desarrollo importante de los glúteos (mayor y menor), los cuales se involucran en diversos movimientos de la pierna y en la postura erguida del tronco, ligados principalmente a la deambulación (Steen y Lane 1998). Además, los coxales muestran un desarrollo del oblicuo externo del abdomen, asociado a la rotación y flexión del tronco.

\subsection{Salud del Individuo 2}

El aparato masticatorio del Individuo 2 presenta una significativa pérdida dental antemortem, para la cual variables como la enfermedad periodontal, la presencia de abscesos, las caries y las respuestas fisiológicas al estrés del uso dental, habrían jugado un rol importante (Clarke y Hirsch 1991; Hillson 1996, 2000; Lukcas 1989). La masiva remodelación de la estructura alveolar impide identificar una o varias de estas variables en detrimento de las otras como explicación causal de la masiva pérdida dental antemortem.

Por último, reacciones periósticas como la registrada en la tibia derecha del Individuo 2 (unilaterales, locales, sin formación de cloacas y en zonas donde el hueso se encuentra cerca de la piel) son comúnmente causadas por traumas locales (Ortner 2003; Waldron 2009). En estos casos, al no tener los estímulos constantes de una infección, los depósitos óseos tienden a formarse como hueso compacto bien organizado y de pequeñas dimensiones, resultando en una osificación perióstica localizada (Ortner 2008; Van der Merwue et al. 2010). Ya que usualmente este proceso finaliza con la incorporación del tejido óseo nuevo al cortical original, dejando únicamente visible una masa o bulto de hueso compacto (Lovell 2008; Ortner 2008), es posible que el impacto haya sido sobre la cara medial y que el individuo murió durante el proceso de osificación del hematoma. Además, considerando la ausencia de indicadores de violencia y el intenso estrés mecánico al que fue sometido el cuerpo de este individuo a 
lo largo del tiempo, es probable que la reacción sea una respuesta a un trauma sufrido durante la realización de tareas cotidianas.

\section{Consideraciones finales}

El sitio Chimpay representa un contexto mortuorio de fines de siglo XIX en el que se inhumaron dos individuos. El contexto en general y el acompañamiento funerario, constituido por objetos indígenas e hispano-criollos, constituyen indicadores de cierta jerarquía: en el caso del Individuo 1 por portar un uniforme militar y en el caso del Individuo 2 por la características y abundancia de su ajuar (Prates et al. 2016).

La configuración morfológica dental del Individuo 1 indica una filiación biológico-poblacional indígena y, si bien en el Individuo 2 no fue posible evaluar los rasgos dentales, las características contextuales sugieren una adscripción cultural indígena. Considerando la importancia de confirmar la ancestría de ambos individuos y validar las inferencias realizadas a partir de indicadores morfológicos y culturales, se están realizando actualmente análisis de ADN antiguo a través de marcadores uniparentales. Con la información disponible hasta el momento es razonable plantear que el Individuo 1 gozó de cierta jerarquía política y que portó un cargo militar del Estado Nacional por su labor como interlocutor diplomático con las parcialidades indígenas. Después de su muerte estos personajes se sepultaban generalmente vistiendo sus uniformes (véase discusión en Prates et al. 2016). En el caso del Individuo 2 no es posible todavía explicar las implicaciones de su presencia en el contexto mortuorio.

En el Individuo 1 los indicadores de estrés mecánico (alteraciones patológicas en la columna vertebral y desarrollo muscular en los miembros inferiores) y el contexto socio-histórico sugieren la realización frecuente de actividades a caballo. En el Individuo 2, aunque el sexo y la edad probablemente desempeñaron un rol importante en su desgaste articular y cambios entésicos, es posible que hayan influido también ciertas actividades cotidianas realizadas sistemática y exclusivamente por las mujeres en la sociedad mapuche (e.g. molienda e hilado; Olea 2010). En lo que respecta a la salud bucal, ambos individuos presentan grandes cantidades de cálculo producto de una deficiente higiene dental. En el caso del hombre, estos depósitos no habrían generado deterioros importantes, mientras que en la mujer, aunque no es posible precisar una causalidad específica a la masiva pérdida dental, es probable que el cálculo haya tenido un rol activo. Ninguno de los dos registra otras lesiones de orden patológico además de las mencionadas. Tampoco se detectan indicadores certeros de sus causas de muerte ni de eventos de violencia interpersonal que permitan comprender con mayor detalle este contexto funerario.

Para finalizar, los resultados discutidos en este trabajo aportan información novedosa sobre la complejidad de las relaciones e interacciones entre indígenas y criollos a finales del siglo XIX en el noreste de Patagonia. La presencia de un indígena con uniforme militar es un correlato directo de los procesos de apropiación y resignificación de la materialidad cultural hispano-criolla por parte de las sociedades indígenas, como consecuencia de varios siglos de contacto y sometimiento (Prates et al. 
2016). Entre los principales aspectos aún pendientes de estudio pueden mencionarse la incorporación de los análisis moleculares para determinar con mayor precisión la ancestría de ambos individuos y la definición a partir del registro etnohistórico y arqueológico de este complejo ritual mortuorio.

Agradecimientos: Deseamos expresar nuestro agradecimiento al Sr. Daniel Cabaza, director del Museo Paleontológico Municipal de Lamarque, por su insustituible y comprometida participación desde el hallazgo del sitio. Al Sr. Tranquilo Sacaría, propietario del Establecimiento Alcarpa S.A, por su buena disposición para que se realicen los trabajos dentro de su propiedad. Al Hospital Escuela-Servicio de Radiología «Médica Veterinaria Miriam Elizabet Villanueva». A la Licda. Alexandrina Amorim Alves, investigadora de la Universidad de Coimbra, Portugal, por sus comentarios y opiniones sobre reacciones periósticas. Este trabajo no habría podido realizarse sin las gestiones y aportes de Antonia Peronja desde la Secretaría de Cultura de la provincia de Río Negro. Estas investigaciones fueron realizadas con fondos de código PICT-2012-0242 y PIP-CONICET Nº 338/10.

\section{Referencias bibliográficas}

Bollini, Gabriel A., Carlos D. Rodríguez-Flórez, Sonia E. Colantonio y Marta G. MÉNDEZ

2006 «Morfología dental de una serie prehistórica de araucanos provenientes de La Patagonia (Argentina)». Boletín de Antropología Universidad de Antioquia 20 (37): 206-226.

BOLM-AUdORFF, Ulrich

1992 «Intervertebral Disc Disorders Due to Lifting and Carrying Heavy Weights». Medical Orthopedic Technology 112: 293-296.

BRIDGES, Patricia S.

1992 «Prehistoric Arthritis in the Americas». Annual Review of Anthropology 21: 67-91.

BRothWELL, Don R.

1981 Digging Up Bones. Nueva York: Cornell University Press.

Buikstra Jane y Douglas H. UbELAKER, eds.

1994 Standards for Data Collection from Human Skeletal Remains. Fayetteville: Arkansas Archaeological Survey Research Series 44.

CANE, Scott

1989 «Australian Aboriginal Seed Grinding and its Archaeological Record: A Case Study from the Western Desert», en The Evolution of Plant Exploitation, David R. Harris y Gordon C. Hillman, eds., pp. 99-119. Londres: Unwin Hyman.

CAPAsso, Luigi, Kenneth A. R. Kennedy y Cynthia A. WilczaK

1999 Atlas of Occupational Markers on Human Remains. Journal of Paleopathology. Monographic Publications 3. Teramo: Edigrafial.

Clarke, Nigel G. y Robert S. Hirsch

1991 «Physiological, Pulpal, and Periodontal Factors Influencing Alveolar Bone», en Advances in Dental Anthropology, Marc A. Kelley y Clark S. Larsen, eds., pp. 241-266. Nueva York: Willey-Liss. 
Devoto, Francisco C. H., Norberto H. Arias, Susana Ringuelet y Néstor H. Palma

1968 «Shovel-Shaped Incisors in a Northwestern Argentine Population». Journal of Dental Research 47 (5): 820-823.

EDYNAK, Gloria Jean

1976 «Lifestyles from Skeletal Material: A Medieval Yugoslav Example», en The Measures of Man, Eugene Giles y Jonathan S. Friedlaender, eds., pp. 408-432. Cambridge: Peabody Museum Press.

Eshed, Vered, Avi Gopher, Ehud GaliLi e Israel HershKovitz

2004 «Musculoskeletal Stress Markers (MSM) in Natufian Hunter-Gatherers and Neolithic Farmers in the Levant: The Upper Limb». American Journal of Physical Anthropology 123 (4): 303-315.

Freivals, Andris

2004 Biomechanics of the Upper Limbs: Mechanics, Modeling, and Musculoskeletal Injuries. Boca Raton: CRC Press.

GALERA, Virginia y Ma Dolores GARRALDA

1993 «Enthesopathies in a Spanish Medieval Population. Anthropological, Epidemiological and Ethnohistorical Aspects». International Journal of Anthropology 8 (4): 247-258.

GonzÁLEZ, Alberto Rex

1979 «Las exequias de Painé Guor». Runa. Archivo para las ciencias del hombre XIII: 137-162.

Guatelli-Steinberg, Debbie, Joel D. Irish y John R. Lukacs

2001 «Canary Islands-North African Population Affinities: Measures of Divergence Based on Dental Morphology». Homo 52 (2): 173-188.

HADLER, Nortin M.

1977 «Industrial Rheumatology: Clinical Investigations into the Influence of Patterns of Usage on the Pattern of Regional Musculo-Skeletal Disease». Arthritis and Rheumatism 20 (4): 1019-1025.

HANIHARA, Kazuro

1968 «Mongoloid Dental Complex in the Permanent Dentition», en Actas del VIII ${ }^{\text {th }}$ International Congress of Anthropological and Ethnological Sciences, pp. 298-300. Tokyo y Kyoto: Science Council of Japan.

Hawkey, Diane E. y Charles F. Merbs

1995 «Activity-Induced Musculoskeletal Stress Markers (MSM) and Subsistence Strategy Among Ancient Hudson Bay Eskimos». International Journal of Osteoarchaeology 5 (4): 324-338.

Hessler, Christian, Helen S. StOhrer, Jurgen MAderT y Klaus Püschel

2012 «Spine Fractures Caused by Horse Riding-Related Accidents». International SportMed Journal 13 (4): 153-160.

Hillson, Simon

1996 Dental Anthropology. Cambridge: Cambridge University Press.

2000 «Dental Pathology», en Biological Anthropology of the Human Skeleton, M. Anne Katzenberg y Shelley R. Saunders, eds., pp. 249-285. Nueva York: Wiley-Liss. 
Hodges, Denise C.

1991 «Temporomandibular Joint Osteoarthritis in a British Skeletal Population». American Journal of Physical Anthropology 85 (4): 367-377.

HRDLICKA, Aleš

1920 «Shovel-Shaped Teeth». American Journal of Physical Anthropology 3 (4): 42965.

JURMAIN, Robert

1980 «The Pattern of Involvement of Appendicular Degenerative Joint Disease». American Journal of Physical Anthropology 53 (1): 143-150.

1990 «Paleoepidemiology of a Central California Prehistoric Population from CAALA-329: II. Degenerative Disease». American Journal of Physical Anthropology 83 (1): 83-94.

1999 Stories from the Skeleton. Behavioral Reconstruction in Human Osteology. Amsterdam: Gordon and Breach.

Jurmain, Robert, Francisca Alves Cardoso, Charlotte Henderson y Sébastien Villotte

2012 «Bioarchaeology's Holy Grail: The Reconstruction of Activity», en Companion to Paleopathology, Anne L. Grauer, ed., pp. 531-552. Oxford: Wiley/Blackwell.

KENNEDY, Kenneth A.R.

1989 «Skeletal Markers of Occupational Stress», en Reconstruction of Life from the Skeleton, Mehmet Y. Işcan y Kenneth A.R. Kennedy, eds., pp. 129-160. Nueva York: Alan R. Liss.

KNÜSEL, Christopher

2000 «Bone Adaptation and its Relationships to Physical Activity in the Past», en $\mathrm{Hu}$ man Osteology in Archaeology and Forensic Sciences, Margaret Cox y Simon Mays, eds., pp. 381-402. Londres: Greenwich Medical Media Ltd.

Kostick, Evan L.

1963 «Facets and Imprints on the Upper and Lower Extremities of Femora from a Western Nigerian Population». Journal of Anatomy 97 (3): 393-402.

LAI, Ping y Nancy C. Lovell

1992 «Skeletal Markers of Occupational Stress in the Fur Trade: A Case Study from a Hudson's Bay Company Fur Trade Post». International Journal of Osteoarchaeo$\operatorname{logy} 2$ (3): 221-234.

LARSEN, Clark S.

2000 Bioarchaeology. Interpreting Behavior from the Human Skeleton. Cambridge: Cambridge University Press.

Lieverse, Angela R., Andrzej W. Weber, Vladimir I. Bazalisskir, Olga I. Goriunova y Nikolai A. SAVEL'EV

2007 «Osteoarthritis in Siberia's Cis-Baikal: Skeletal Indicators of Hunter-Gatherer Adaptation and Cultural Change». American Journal of Physical Anthropology 132 (1): $1-16$.

Lovejoy, C. Owen, Richard S. Meindl, Thomas R. Pryzbeck y Robert P. Mensforth 1985 «Chronological Metamorphosis of the Auricular Surface of the Ilium: A New Method for the Determination of Adult Skeletal Age at Death». American Journal of Physical Anthropology 68 (1): 15-28. 
LOVELL, Nancy C.

2008 «Analysis and Interpretation of Skeletal Trauma», en Biological Anthropology of the Human Skeleton, M. Anne Katzenberg y Shelley R. Saunders, eds., pp. 341386. Nueva York: Wiley-Liss.

LUKACS, John R.

1989 «Dental Paleopathology: Methods for Reconstructing Dietary Patterns», en Reconstruction of Life from the Skeleton, Mehmet Y. Işcan y Kenneth A.R. Kennedy, eds., pp. 261-286. Nueva York: Alan R. Liss, Inc.

MANDRINI, Raúl

2008 La Argentina aborigen. De los primeros pobladores a 1910. Buenos Aires: Siglo XXI.

ManN, Robert W. y Sean P. MurPhy

1990 Regional Atlas of Bone Disease. A Guide to Pathologic and Normal Variation in the Human Skeleton. Springfield: Charles C. Thomas Publisher

Martin, Cecil P.

1932 «Some Variations in the Lower End of the Femur Which Are Especially Relevant in the Bones of Primitive People». Journal of Anatomy 66 (3): 371-383

Merbs, Charles F.

1983 Patterns of Activity-Induced Pathology in a Canadian Inuit Population. Ottawa: Archaeological Survey of Canada.

Molleson, Theya

1994 «The Eloquent Bones of Abu Hureya». Scientific American 271 (2): 70-75.

2007 «A Method for the Study of Activity Related Skeletal Morphologies». Bioarchaeology of the Near East 1: 5-33.

Molnar, Petra, Torbjorn P. Ahlstrom e Ido Leden

2011 «Osteoarthritis and Activity: An Analysis of the Relationship Between Eburnation, Musculoskeletal Stress Markers (MSM) and Age in two Neolithic HunterGatherer Populations from Gotland, Sweden». International Journal of Osteoarchaeology 21 (3): 283-291.

MoLnAR, Stephen

1971 «Human Tooth Wear, Tooth Function and Cultural Variability». American Journal of Physical Anthropology 34 (2): 175-188.

Murillo Rodríguez, Silvia

2002 La vida a través de la muerte: estudio biocultural de las costumbres funerarias en el Temazcaltepec prehispánico. Madrid: Plaza y Valdés editores.

NacuzzI, Lidia Rosa

1998 Identidades impuestas. Tehuelches, aucas y pampas en el norte de la Patagonia. Buenos Aires: Sociedad Argentina de Antropología.

Neves, Walter A.

1984 «Estilo de Vida e osteobigrafia: a reconstituição do comportamento pelos osso humanos». Revista Pré-História 6: 287-291.

OGDen, Alan R.

2008 «Advances in the Paleopathology of Teeth and Jaws», en Advances on Human Paleopathology, Ron Pinhasi y Simon Mays, eds., pp. 283-308. West Sussex: John Wiley \& Sons. 
Olea Rosenbluth, Catalina

2010 La mujer en la sociedad mapuche. Siglos XVI a XIX. Santiago de Chile: Servicio Nacional de la Mujer.

ORTNER, Donald J.

2003 Identification of Pathological Conditions in Human Skeletal Remains. San Diego: Elsevier.

2008 «Differential Diagnosis of Skeletal Lesions in Infectious Disease», en Advances on Human Paleopathology, Ron Pinhasi y Simon Mays, eds., pp. 191-214. West Sussex: John Wiley \& Sons.

Pfirrmann, Christian W. y Donald Resnick

2001 «Schmorl Nodes of the Thoracic and Lumbar Spine: Radiographic-Pathologic Study of Prevalence, Characterization, and Correlation with Degenerative Changes of 1,650 Spinal Levels in 100 Cadavers». Radiology 219 (2): 368-374.

Powell, Mary Lucas

1985 «The Analysis of Dental Caries and Wear for Dietary Reconstruction», en The Analysis of Prehistoric Diets, Robert I. Gilbert y James H. Mielke, eds., pp. 307338. Orlando: Academic Press.

Prates, Luciano y Violeta Di Prado

2013 «Sitios con entierros humanos y ocupaciones residenciales en la cuenca del río Negro (Norpatagonia, Argentina). Diacronía y multicausalidad». Latin American Antiquity 24 (4): 451-466.

Prates, Luciano, Alejandro Serna, Emiliano Mange e Ingrid de Jong

2016 «Expresión material de la interacción entre indígenas y criollos en un sitio funerario del siglo XIX de Norpatagonia (sitio Chimpay, Río Negro)». Intersecciones en Antropología 17 (1): 35-48.

RANDO, Carolyn y Tony WALDRON

2012 «TMJ Osteoarthritis: A New Approach to Diagnosis». American Journal of Physical Anthropology 148 (1): 45-53.

REINHARD, Karl J.

1993 «Trade, Contact, and Female Health in Northeast Nebraska», en In the Wake of Contact. Biological Responses to Conquest, Clark S. Larsen y George R. Milner, eds., pp. 63-74. Nueva York: Wiley Liss.

Rodrigues Carvalho, Claudia

2004 Marcadores de estresse ocupacional em populações sambaquieiras do Litoral Fluminense. Tesis doctoral inédita, Escola Nacional de Saúde Pública/Fundação Oswaldo Cruz, Río de Janeiro.

ScOTt, G. Richard

1980 «Populations Variation of Carabelli's Trait». Human Biology 52 (1): 63-78.

Scott, G. Richard y Christy G. TURNER

2000 The Anthropology of Modern Human Teeth. Cambridge Studies in Biological and Evolutionary Anthropology 20. Cambridge: Cambridge University Press.

SMITH, B. Holly

1984 «Patterns of Molar Wear in Hunter-Gatherers and Agriculturalists». American Journal of Physical Anthropology 63 (1): 39-56. 
SPERBER, Geoffrey H.

2004 «The Genetics of Odontogenesis: Implications in Dental Anthropology and Palaeodontology». Dental Anthropology 17: 1-7.

SteEn, Susan L. y Robert W. LANe

1998 «Evaluation of Habitual Activities Among Two Alaskan Eskimo Populations Based on Musculoskeletal Stress Markers». International Journal of Osteoarchaeo$\operatorname{logy} 8$ (5): 341-353.

TrinKaUs, Erik

1975 «Squatting Among the Neanderthals: A Problem in the Behavioral Interpretation of Skeletal Morphology». Journal of Archaeological Science 2: 327-351.

Turner II, Christy, Christian Nichol y G. Richard ScotT

1991 «Scoring Procedures for Key Morphological Traits of the Permanent Dentition: The Arizona State University Dental Anthropology System», en Advances in Dental Anthropology, Marc A. Kelley y Clark S. Larsen, eds., pp. 13-31. Nueva York: Wiley-Liss.

UBELAKER, Douglas H.

1979 «Skeletal Evidence for Kneeling in Prehistoric Ecuador». American Journal of Physical Anthropology 51 (4): 679-686.

1982 Human Skeletal Remains. Excavation, Analysis, Interpretation. Washington: Taraxacum.

ÜSTÜNDAG, Handan y Abdullah DEvecI

2011 «A Possible Case of Scheuermann's Disease from Akarçay Höyük, Birecik (Şanliurfa, Turkey)». International Journal of Osteoarchaeology 21(2): 187-196.

Van Der Merwe, Alie E., George MaAt y Maryna Steyn

2010 «Ossified Haematomas and Infectious Bone Changes on the Anterior Tibia: Histomorphological Features as an Aid for Accurate Diagnosis». International Journal of Osteoarchaeology 20 (2): 227-239.

Villotte, Sébastien, Dominique Castex, Vincent Couallier, Olivier Dutour, Christopher

J. KNÜSEl y Dominique Henry-GAMbIER

2010 «Enthesopathies as Occupational Stress Markers: Evidence from the Upper Limb». American Journal of Physical Anthropology 142 (2): 224-234.

WALDRON, Tony

2009 Paleopathology. Cambridge: Cambridge University Press.

WeISS, Elizabeth y Robert JURMAIN

2007 «Osteoarthritis Revisited: A Contemporary Review of Aetiology». International Journal of Osteoarchaeology 17 (5): 437-450.

Wentz, Rachel K. y Nancy T. De GRummond

2009 «Life on Horseback: Palaeopathology of Two Scythian Skeletons from Alexandropol, Ukraine». International Journal of Osteoarchaeology 19 (1): 107-115.

White, Tim D. y Pieter A. Folkens

2005 The Human Bone Manual. Londres: Elsevier-Academic Press.

Wilczak, Cynthia A.

1998 «Consideration of Sexual Dimorphism, Age, and Asymmetry in Quantitative Measurements of Muscle Insertion Sites». International Journal of Osteoarchaeology 8 (5): $311-325$. 